Neugebauer, H. E. J. (May 1957), Application of the reciprocity theorem to the calculation of antenna patterns, RCA Victor Res. Rept. 7-601-7.

Ratcliffe, J. A. (1959), The Magneto-Ionic Theory and its Application to the Ionosphere (Cambridge Univ. Press, Cambridge, Mass.).

Richmond, J. H. (1956), Antenna pattern distortion by dielectric sheets, Trans. IRE AP-4, No. 2, $139-142$.

Stix, T. H. (1962), The Theory of Plasma Waves, p. 11 (McGraw.Hill Book Co., Inc., New York, N.Y.).

(Paper 3-9-448)

\title{
lonospheric Focusing for an Antenna in the Presence of the Geomagnetic Field
}

\author{
D. Walsh and Herschel Weil \\ Radio Astronomy Observatory, University of Michigan, Ann Arbor, Mich. 48104, U.S.A. \\ (Received December 1, 1967; revised February 23, 1968)
}

\begin{abstract}
A geometrical optics treatment of ionospheric focusing is presented which is exact within the assumptions of horizontal stratification of the ionosphere and of the collision-free Appleton-Hartree equation. In addition to effects of ray refraction, the expression derived for gain distribution in the beam outside the ionosphere includes in a natural manner the directional characteristics of the antenna in the plasma. These involve both the antenna geometry and the characteristic polarizations of the incident waves. The factors in the gain expression which are dependent only on the plasma characteristics are expressed in closed form. Evaluation of the remaining factors, in general, requires knowledge of the current distribution on the antenna. For the case of an electrically short dipole, this evaluation is carried out and the gain distribution expressed in closed form, apart from a normalizing factor that can only be determined by numerical integration. The properties of the gain distribution are illustrated by contour plots.
\end{abstract}

\section{Introduction}

Refraction by the electron gradients in the ionosphere can have a pronounced effect on the gain pattern, as observed outside the ionosphere, of an antenna located within the ionosphere. This so-called "ionospheric focusing" may occur for radiation either above or below the ionosphere, though previous discussions emphasize the former case as a prospective method for producing a directive beam for low frequency radio astronomy observations from space vehicles. For simplicity, most discussions of the subject neglect the earth's magnetic field (Haselgrove, Haselgrove, and Jennison, 1961; Budden, 196la; Smith, 1961; Jennison, 1961; Daniell, 1964, 1966). Budden and Hugill (1964) discussed the field strengths of cosmic noise signals in the ionosphere taking into account the geomagnetic field, but the only direct attempt at determining the influence of the geomag. netic field on the polar diagram outside the ionosphere appears to be by Papagiannis and Huguenin (1964), who present some very instructive directivity patterns.

The results of Papagiannis and Huguenin suffer from two drawbacks. First, they are difficult to extend to real antennas because they apply, in effect, to antennas uniformly sensitive, within the ionosphere, to flux from any direction. Thus for a real antenna, the results must be multiplied by a term representing its directional sensitivity within the ionosphere. This depends, among other factors, on the characteristic polarizations of the incident waves, which are determined by the local anisotropic plasma and are nowhere taken into account in the calculations. Second, their method involves approximations, even for a hypothetical isotropic antenna, which arise from the analytic representation they adopt for refractive index surfaces. They state that for a given propagation direction it is sufficient to replace the true refractive index surface by a "simple geometric surface with the identical radial distance and gradient in the proper 
direction." In fact, it is also necessary that the surface have the correct Gaussian curvature. This appears to be ignored in. Papagiannis' and Huguenin's crucial equation (7.20), where the parameters $a$ and $b$ are held constant but should actually be treated as functions of their variables $\theta$ and $\varphi$. The magnitude of the error due to this approximation is difficult to estimate analytically.

In this paper we derive a general expression for the distribution of the gain in the beam outside the ionosphere for any antenna. This expression may be evaluated if the current distribution on the antenna when used as a radiator is known. This evaluation is then performed for the case of greatest practical interest, namely, a short electric dipole. The result is valid for any orientation of the dipole and magnetic field and involves no approximation to the refractive index surfaces. We assume that (i) the ionosphere is horizontally stratified, i.e., the electron density and geomagnetic field are functions only of altitude; (ii) collisions may be neglected; (iii) refraction is controlled by the Appleton-Hartree equation; and (iv) ray tracing by geometrical optics is adequate.

In the ionosphere each of the two possible modes of propagation, ordinary and extraordinary, propagates independently with its own characteristic polarization. The energy of a wave entering the ionosphere with arbitrary polarization will be divided naturally between the two modes. Each will give rise to its individual focused pattern, though under certain conditions the antenna may be situated in a region to which only one modé can propagate from outside the ionosphere and then only the corresponding beam will exist. For a particular direction in the beam outside the ionosphere, the characteristic polarization of each mode is determined by the limiting conditions in the ionosphere where it first influences a wave entering from free space. Energy in a wave with one of these characteristic polarizations which propagates to the antenna from outside the ionosphere may do so by two distinct paths, one "direct" and one "reflected," with a corresponding phase difference. Thus, for a given direction in the beam outside the ionosphere, a total of four waves may be incident at the antenna from different directions and with phase differences depending on the propagation paths. Due regard must be paid to these phase differences if the fine structure of the total response of the antenna is of interest. These phase differences will not be considered further in what follows.

\section{General Formula for Gain}

We consider a plane wave originating outside the ionosphere and incident at the antenna in one or another of the magnetoionic modes. Quantities evaluated outside the ionosphere and at the antenna will be indicated by subscripts 0 and $A$, respectively. Thus the Poynting vector has magnitude $S_{0}$ and zenith angle $\mathrm{X}_{0}$ outside the ionosphere and magnitude $S_{A}$ and zenith angle $\mathrm{X}_{A}$ at the antenna. Since the vertical component of Poynting flux has constant magnitude in a horizontally stratified ionosphere,

$$
\left|S_{0} \cos \mathrm{X}_{0}\right|=\left|S_{A} \cos \mathrm{X}_{A}\right| .
$$

Following the discussion of antennas in magnetoionic media given by Weil and Walsh (1967) (hereafter referred to as paper I), the collecting area associated with the direction of propagation of the wave at the antenna is $(I, 4.5)$

$$
A_{A}=|V|^{2} /\left(8 R S_{A}\right),
$$

where $V$ is the open-circuit voltage induced in the antenna and $R$ is its radiation resistance in the plasma. (The number in parentheses above this expression indicates its number in paper $\mathrm{I}$; this notation will be used when equations from paper I are used in the present paper.) The collecting area associated with the beam outside the ionosphere is

$$
A_{0}=|V|^{2} /\left(8 R S_{0}\right) \text {. }
$$

Combining (2.1), (2.2), and (2.3) gives

$$
\frac{A_{0}}{A_{A}}=\frac{S_{A}}{S_{0}}=\left|\frac{\cos \mathrm{X}_{0}}{\cos \mathrm{X}_{A}}\right| \text {. }
$$

This simple result is the key to deriving expressions for the collecting area and gain in the beam outside the ionosphere. It is convenient to rewrite $A_{A}$ with the aid of paper $I(I, 4.18)$,

$$
A_{A}=\zeta_{0}|h|^{2} /(4 m R) .
$$

In (2.5), $\zeta_{0}$ is the impedance of free space. The quantity $m$ was introduced in paper I such that the time-average Poynting flux at the antenna in a sinusoidal plane wave with electric field $\vec{E}$ is $m|E|^{2}\left(2 \zeta_{0}\right)$; for an isotropic medium, $m$ reduces to the refractive index, but for the anisotropic plasma it is a function of the wave-normal direction. The quantity $|h|$ is the magnitude of the vector effective length, defined for anisotropic media in paper I; it is, of course, a function of the antenna geometry as well as wave-normal direction. Finally, combining the last two equations with the fact that outside the ionosphere the gain is $G_{0}=4 \pi A_{0} / \lambda_{0}^{2}$, it follows that

$$
G_{0}=\frac{\pi \zeta_{0}}{\lambda_{0}^{2}} \cdot \frac{|h|^{2}}{R} \cdot\left|\frac{\cos \mathrm{X}_{0}}{m \cos \mathrm{X}_{A}}\right|,
$$

where $\lambda_{0}$ is the free-space wavelength.

Expression (2.6) is written as the product of three terms, the first of which involves only constants. The last term is independent of the particular antenna and is considered further in the next section. The remaining term, $|h|^{2} / R$, is the only one involving the current distribution of the antenna; if this distribution is known, 
both $|h|$ and $R$ may be found as outlined in paper $I$. It may be emphasized that while $|h|$ is a function of wave-normal direction, $R$ is not; $R$ has the nature of a normalizing constant, and the ratio of $G_{0}$ for two different values of $X_{0}$ may be calculated without knowledge of $R$. As discussed in paper I, there are two options in the definition of $R$ in the above equations: (i) $R$ may be the total radiation resistance; (ii) it may be the portion of the radiation resistance associated with only one of the magnetoionic modes. The gain integrated over the solid angle of the beam is $4 \pi$ in both cases, provided the integration includes both magnetoionic modes if option (i) is adopted, but includes only the mode associated with $R$ if option (ii) is adopted. Which option is more useful depends on the particular application. For radio astronomy applications, option (ii) has some advantages.

\section{3.: Development of Gain Formula}

Equation (2.6) is of general application to any antenna. While the prime interest is the relationship of $G_{0}$ to $\mathrm{X}_{0}$, it is convenient to retain the wave-normal direction at the antenna as an independent parameter and to express both $G_{0}$ and $\mathrm{X}_{0}$ in terms of it. The factor $\left|\cos \mathrm{X}_{0} / m \cos \mathrm{X}_{A}\right|$ in (2.6) cannot be evaluated without further manipulation; in this section, with the aid of standard magnetoionic theory, it will be expressed explicitly as a function of the wave-normal direction and the plasma and gyrofrequencies at the antenna. In doing so, some quantities will deliberately not be written in what superficially may appear to be their simplest forms in an effort to remove, as far as possible, sources of indeterminacies that may be troublesome when carrying out numerical computations.

\subsection{Notation and Geometry}

The geometry of the problem is shown in figure 1 , where the various points represent the intersections of vectors with an infinite sphere, and the curves are arcs of great circles. The angles subtended at the center by various arcs are indicated. We use spherical polar coordinates with the vertical as polar direction, $\chi$ representing zenith angle, and $\tau$ azimuthal angle measured

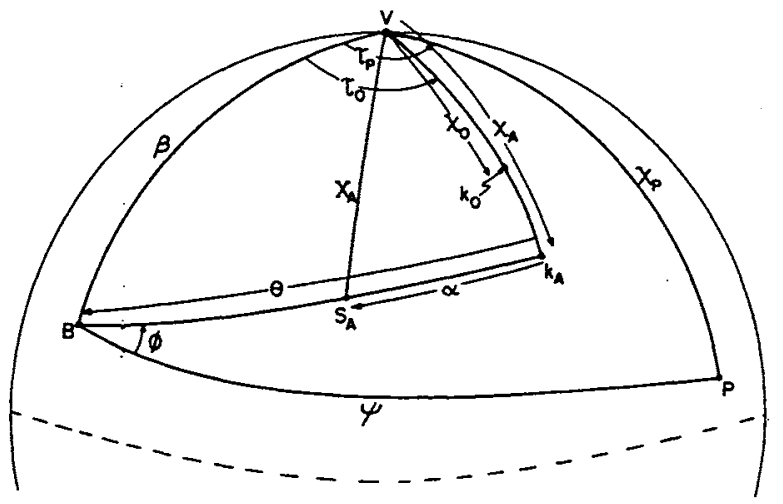

FIGURE 1. Geometry for gain calculation. from the magnetic meridian plane. Quantities shown in figure 1 and not previously defined are as follows:

$V=$ vertical.

$B=$ direction of earth's magnetic field.

$\beta=$ angle between $\vec{B}$ and vertical, $0 \leqslant \beta \leqslant \pi$.

$k_{0}=$ direction of wave normal outside ionosphere, coordinates $\chi_{0}, \tau_{0}$.

$k_{A}=$ direction of wave normal at antenna, coordinates $\chi_{A}, \tau_{0}$.

$\theta=$ angle from $\vec{k}_{A}$ to $\vec{B} ; 0 \leqslant \theta \leqslant \pi$.

$S_{A}=$ direction of Poynting vector at antenna.

$\alpha=$ angle from $\vec{k}_{A}$ to $\vec{S}_{A} ;-\pi / 2<\alpha<\pi / 2$ (positive if in same sense as $\theta$ ).

In addition, certain quantities associated with an electric dipole are included in figure 1 and in this discussion of the geometry, though they are not required until section 4 . They are:

$$
\begin{aligned}
& p=\text { direction of dipole, coordinates } \chi_{p}, v_{p} . \\
& \psi=\text { angle between } \vec{p} \text { and } \vec{B} ; 0<\psi<\pi . \\
& \varphi=\text { angle between planes containing }(\vec{B}, \vec{p}) \text { and } \\
& \qquad\left(\vec{B}, \vec{k}_{A}\right) ; 0 \leqslant \varphi \leqslant 2 \pi .
\end{aligned}
$$

In constructing figure 1 , use has been made of the well-known facts that $\vec{k}_{0}$ and $\vec{k}_{A}$ are in the same vertical plane and that $\vec{B}, \vec{k}_{A}$, and $\vec{S}_{A}$ are coplanar. Outside the ionosphere, the Poynting vector has the same direction as the wave normal, hence $X_{0}=\chi_{0}$.

$G_{0}$ is required in terms of parameters related to the antenna geometry (for the electric dipole, these will include $\chi_{p}$ and $\tau_{p}$ ) together with $\beta, \chi_{A}$, and $\tau_{0}$. It is thus necessary to express a number of the other angles in terms of these quantities. By straightforward spherical trigonometry we find:

$$
\begin{aligned}
& \cos \psi=\cos \beta \cos \chi_{p}+\sin \beta \sin \chi_{p} \cos \tau_{p}, \\
& \cos \theta=\cos \beta \cos \chi_{A}+\sin \beta \sin \chi_{A} \cos \tau_{0},
\end{aligned}
$$

and

$$
\varphi=\widehat{V B P}-\widehat{V B k_{A}} \text {, }
$$

where

$$
\begin{aligned}
& \cos \widehat{V B p}=\left(\sin \beta \cos \chi_{p}-\cos \beta \sin \chi_{p} \cos \tau_{p}\right) / \sin \psi, \\
& \sin \widehat{V B p}=\sin \chi_{p} \sin \tau_{p} / \sin \psi, \\
& \cos \widehat{V B k_{A}}=\left(\sin \beta \cos \chi_{A}-\cos \beta \sin \chi_{A} \cos \tau_{0}\right) / \sin \theta, \\
& \sin \widehat{V B k_{A}}=\sin \chi_{A} \sin \tau_{0} / \sin \theta .
\end{aligned}
$$

Both sine and cosine of $\widehat{V B p}$ and $\widehat{V B k_{A}}$ are needed, since these angles are in the range 0 to $2 \pi$. Also, starting with

$\cos \mathrm{X}_{A}=\cos \beta \cos (\theta-\alpha)+\sin \beta \sin (\theta-\alpha) \cos \widehat{V B k_{A}}$, 
a little manipulation yields the result:

$\cos \mathrm{X}_{A} / \cos \alpha=\cos \chi_{A}+\left(\cos \beta-\cos \chi_{A} \cos \theta\right) \tan \alpha / \sin \theta$,

which will be used later.

Parameters characteristic of the ionospheric plasma at the antenna are:

$$
\begin{aligned}
& X=\text { (local plasma frequency })^{2} /(\text { wave frequency })^{2} \text {, } \\
& Y=(\text { local gyrofrequency)/(wave frequency). }
\end{aligned}
$$

Then, defining

$$
\eta=\frac{Y \sin ^{2} \theta}{2(1-X)} \mp\left[\frac{Y^{2} \sin ^{4} \theta}{4(1-X)^{2}}+\cos ^{2} \theta\right]^{1 / 2},
$$

( - sign for ordinary, + for extraordinary mode), the Appleton-Hartree formula for refractive index at the antenna, $n$, becomes

$$
n^{2}=1-X /(1-\eta Y) .
$$

Another useful quantity is

$$
\xi=\eta Y\left(n^{2}-1\right) /(1-X) .
$$

It is convenient to introduce a right-handed, orthogonal $x, y, z$ system, in which the $z$ axis is parallel to $\vec{k}_{A}$ and the $x$ axis is chosen such that $\vec{B}$ lies in the $x z$ plane. In this system the direction cosines of $\vec{p}$, i.e., the components of unit vector $\overrightarrow{\hat{p}}$, are

$$
\begin{aligned}
& p_{x}=\cos \psi \sin \theta-\sin \psi \cos \theta \cos \varphi \\
& p_{y}=\sin \psi \sin \varphi \\
& p_{z}=\cos \psi \cos \theta+\sin \psi \sin \theta \cos \varphi .
\end{aligned}
$$

\subsection{Application to Gain Formula}

It is now straightforward to apply several results known from the theory of propagation in a magnetoionic medium to the general gain formula (2.6). First we note that for a horizontally stratified medium, Snell's law is

$$
\sin \chi_{0}=n \sin \chi_{A} .
$$

This relationship is independent of the vertical distribution of electron density. Since $n$ is a fairly complicated function of, among other quantities, $\chi_{A}$, it is difficult to solve (3.7) for $\chi_{A}$ if given $\chi_{0}$. This is why $\chi_{A}$ is preferred as independent parameter.

Next we use the result from paper I (I, A.10; A.11):

$$
m=\frac{n}{\cos \alpha} \cdot \frac{\left|E_{x}\right|^{2}+\left|E_{y}\right|^{2}}{\left|E_{x}\right|^{2}+\left|E_{y}\right|^{2}+\left|E_{z}\right|^{2}},
$$

where $E_{x}, E_{y}, E_{z}$ are components of the electric field
$\vec{E}$ in a plane wave of arbitrary amplitude with the given wave normal $\vec{k}_{A}$. The components of $\vec{E}$ are related by:

$$
\begin{aligned}
& E_{y} / E_{x}=-i \eta / \cos \theta, \quad(i=\sqrt{-1}) \\
& E_{z} / E_{x}=\xi \sin \theta / \cos \theta .
\end{aligned}
$$

(Budden, 1961b, pp. 48-54). Using (3.8) and (3.9) we obtain

$$
\frac{\cos \mathrm{X}_{0}}{m \cos \mathrm{X}_{d}}=\frac{\cos \chi_{0}}{n\left(\cos \mathrm{X}_{A} / \cos \alpha\right)} \cdot \frac{\cos ^{2} \theta+\eta^{2}+\xi^{2} \sin ^{2} \theta}{\cos ^{2} \theta+\eta^{2}} .
$$

The factor $\left(\cos \mathrm{X}_{A} / \cos \alpha\right)$ in the denominator of (3.10) may be evaluated by using (3.4) together with the result

$$
\tan \alpha=-\xi \cos \theta \sin \theta /\left(\cos ^{2} \theta+\eta^{2}\right)
$$

(Budden, 196lb, p. 248), thereby completely eliminating $\alpha$ from the computation. Inserting (3.10) in (2.6) we have the general gain formula,

$G_{0}=\frac{\pi \zeta_{0}}{\lambda_{0}^{2}} \cdot \frac{|h|^{2}}{R}$

$\cdot\left|\frac{\cos \chi_{0}\left(\cos ^{2} \theta+\eta^{2}+\xi^{2} \sin ^{2} \theta\right)}{n\left[\cos \chi_{A}\left(\cos ^{2} \theta+\eta^{2}\right)-\xi \cos \theta\left(\cos \beta-\cos \chi_{A} \cos \theta\right)\right]}\right|$

The trigonometric function enclosed in absolute value signs is now a function only of $X, Y, \beta, \chi_{A}$, and $\tau_{0}$ if use is made of various subsidiary expressions elsewhere in this section.

\section{Gain of a Short Electric Dipole}

To apply (3.12) to a particular antenna, the factor $|h|^{2} / R$ must be evaluated. In general, this requires knowledge of the current distribution on the antenna, which is, unfortunately, not available for many cases. For a short electric dipole, however, $|h|^{2} / R$ can be evaluated. This is of greatest practical importance, since at the frequencies where focusing may be used, namely a few megahertz per second, antennas on space vehicles are usually small compared with the wavelengths.

\subsection{Derivation of Gain Formula}

Consider a center-fed, linear dipole of length $2 a\left(\ll \lambda_{0}\right)$ with current distribution $I(p) \overrightarrow{\hat{p}}$ when used as a radiator. When used to receive radiation of one characteristic mode through the focused beam, the wavelength for any $\vec{k}_{A}$ associated with this mode is greater than $\lambda_{0}$. This is not necessarily so for the other mode of propagation; for example, if the antenna is in a region where $1-Y^{2}<X<1, Y<1$, the refractive 
index for the extraordinary mode may be very large for certain directions and the wavelength correspondingly small. However, extraordinaio waves from outside the ionosphere (where $X=0$ ) cannot reach such a region because they would have to pass through the nonpropagating region $1-Y<X<1-Y^{2}$. Thus, in this case, waves arriving at the antenna from outside the ionosphere are ordinary waves for which $n<1$ at the antenna, and their wavelengths exceed $\lambda_{0}$. For the propagation mode of interest, then, using a result of paper I $(I, 4.26)$,

$$
|h|^{2}=|L|^{2}|\overrightarrow{\hat{p}} \cdot \vec{E}|^{2} /|E|^{2},
$$

where $(I, 4.25)$

$$
L=\frac{1}{I(0)} \int_{-a}^{a} I(p) d p .
$$

Suppose now that $R$ is chosen according to option (ii) discussed in section 2 , that is, $R$ is the component of radiation resistance associated with the propagation mode of interest. Then, as shown in paper I (eq 4.29), $R$ is proportional to $|L|^{2}$. Thus the ratio $|h|^{2} / R$ is independent of $L$, and a knowledge of the current distribution $I(p)$ in the plasma is not necessary for the computation of $G_{0}$. An arbitrary value for $|L|^{2}$ may be adopted, provided it is used in both $|h|^{2}$ and $R$. A convenient choice is the free-space distribution. The free-space radiation resistance is then, using a well-known result $(\mathrm{I}, 4.30)_{3}$

$$
R_{0}=(2 \pi / 3) \zeta_{0}|L|^{2} \lambda{ }^{2}
$$

so we may write:

$$
\frac{\pi \zeta_{0}}{\lambda_{0}^{2}} \cdot \frac{|h|^{2}}{R}=\frac{3}{2\left(R / R_{0}\right)} \cdot \frac{\left|p_{x} E_{x}+p_{y} E_{y}+p_{z} E_{z}\right|^{2}}{\left|E_{x}\right|^{2}+\left|E_{y}\right|^{2}+\left|E_{z}\right|^{2}} .
$$

Finally, using the relations (3.9) in the last equation and substituting the result in (3.12), we have:

$$
\begin{aligned}
& G_{0}=\frac{3}{2\left(R / R_{0}\right)} \\
& \cdot\left|\frac{\cos \chi_{0}\left[\left(p_{x} \cos \theta+\xi p_{z} \sin \theta\right)^{2}+\eta^{2} p_{y}^{2}\right]}{n\left[\cos \chi_{A}\left(\cos ^{2} \theta+\eta^{2}\right)-\xi \cos \theta\left(\cos \beta-\cos \chi_{A} \cos \theta\right)\right]}\right| .
\end{aligned}
$$

Within the limitations of the simplifying assumptions listed in section 1, this expression for the gain of a short dipole is exact. The directional dependance of $G_{0}$ is determined purely by the function enclosed in the absolute magnitude signs and, with the use of subsidiary expressions given earlier in the paper, is in closed form as a function of plasma, gryo- and wave-frequencies and geomagnetic field direction $(X, Y, \beta)$, dipole orientation $\left(\chi_{p}, \tau_{p}\right)$, and wave-normal direction at the antenna $\left(\chi_{A}, \tau_{0}\right)$. Together with Snell's law, $G_{0}$ is determined as a function of $\chi_{0}$.

The normalizing factor involving $R / R_{0}$ cannot, apparently, be determined in closed form. It could be determined, in principle, by the requirement that the integral of $G_{0}$ over the solid angle of the beam be $4 \pi$. However, more convenient integral forms for $R / R_{0}$ exist in the literature, several of which are summarized in paper I. It is interesting to note that the value of $R / R_{0}$ required in (4.5) is to be calculated on the as. sumption that the current distribution $I(p)$ in the plasma is the same as in free space. This is, in fact, the assumption on which most formulas for $R / R_{0}$ are based, so they will lead to correct results when used in (4.5) regardless of the physical validity of this assumption.

\subsection{Illustration and Discussion}

The characteristics of (4.5) are difficult to visualize except by numerical evaluation for specific cases. some examples are given in figures 2 and 3 , which are plots of constant gain contours outside the ionosphere with the vertical as the origin of coordinates and $\chi_{0}$ represented by radial distance. Each plot refers only to "direct" rays $\left(\mathrm{X}_{A} \leqslant 90^{\circ}\right)$ incident on the antenna. For "reflected" rays $\left(\mathrm{X}_{A}>90^{\circ}\right)$ the contours are identical except that the plot must be rotated through $180^{\circ}$ in $\tau_{0}$. The complete polar diagram results from the superposition of these two with due regard to phase. The centroid of the resultant polar diagram is clearly the zenith. The plots were made, for the most part, by a digital plotter and consist of straight-line segments connecting discrete points of constant gain. In most places these points are close enough to give the impression of a smooth curve, but occasionally, usually where the contours have high curvature, the line segments are noticeable and are, of course, artificial. The contours have uniform intervals in $G_{0}$, the highest value plotted being 40 when $X=0.6$ and 100 when $X=0.96$; in addition, the boundary of the beam is plotted in each case. Between the boundary and the next outermost contour there is often a gap in which $G_{0}$ is higher than the highest contour plotted.

The values of $X, Y$, and $\beta$ are the same as used for illustration by Papagiannis and Huguenin (1964) to facilitate comparison with that paper. In each case, $X>1-Y$, so only the ordinary mode can propagate to the antenna from outside the ionosphere. Plots with $Y=0$ are given so that the influence of the magnetic field is clear. Several features merit comment.

(i) The outer boundary of the beam is independent of the antenna. The beamwidth perpendicular to the magnetic meridian plane is unaffected by the field, but it is elongated in the magnetic meridian by an amount depending on $\beta$. This is evident also in the results of Papagiannis and Huguenin (1964), and is a consequence of the directional characteristics of the refractive index at the antenna and Snell's law.

(ii) At the boundary of the beam the gain is infinite 


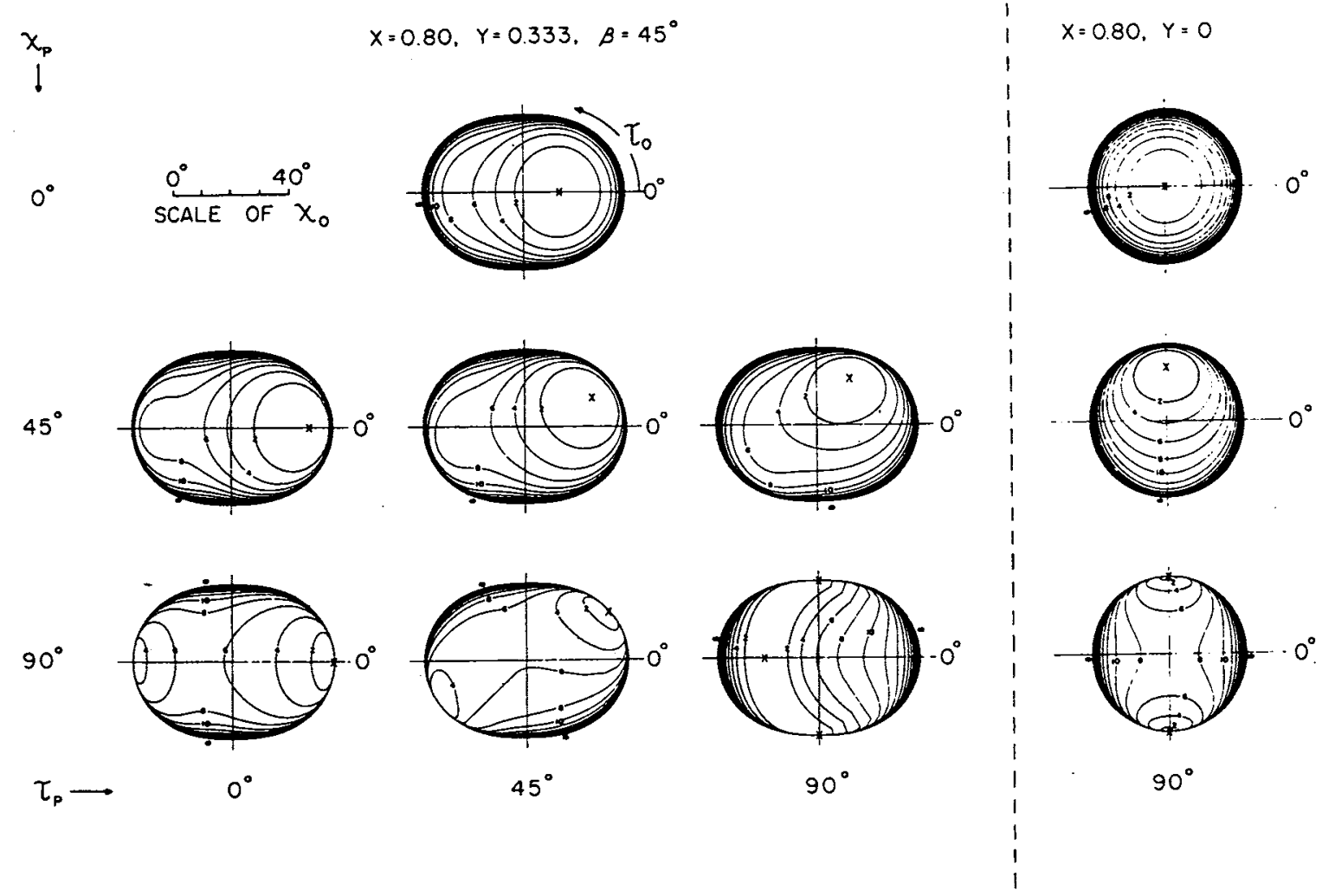

FIGURE 2. Illustrating effect of dipole orientation on gain distribution outside ionosphere for "direct" rays in ordinary mode. Magnetic meridian plane is axis labeled $0^{\circ}$. Constant gain contours are plotted with gain interval 2 up to gain 40, only lowest few contours being labeled; in additien. boundary of beam $($ gain $\infty)$ is plotted. Points of zero gain are indicated by crosses.

(with exceptions discussed in the next paragraph). This is well known for the isotropic case $(Y=0)$, and is a consequence of the use of ray optics to determine field strengths in a region where this type of approximation to the correct solution of the electromagnetic wave problem is invalid. Budden (1961a) shows that a more exact solution yields finite, though large, fields and gain, and that the beam, in fact, extends slightly beyond the sharp boundary given by ray theory. Formally, the infinite gain in the ray theory results because rays at the beam boundary are those which, at the antenna, have just reached their reflection level and are traveling horizontally; the factor $\cos \mathrm{X}_{\mathrm{A}}$ in the denominator of $(2.6)$ is then zero.

(iii) The zero gain points occur when the factor $\vec{p} \cdot \vec{E}$ in $(4.1)$ is zero, i.e., when the incident field is in a plane perpendicular to the dipole. In the isotropic case this occurs when both the incident wave normal and ray are parallel to the dipole. A zero in the anisotropic case corresponds, in general, to neither of these conditions since the plane of $\vec{E}$ is not perpendicular to either wave normal or ray. For the case $\beta=0$ in figure 3 , the gain is zero at the boundary of the beam, rather than at infinity as in all the other cases. In this case, any ray traveling horizontally at the antenna is perpendicular to the geomagnetic field, the wave normal has the same direction as the ray, $\vec{E}$ is linearly polarized in a direction parallel to the geomagnetic field, i.e., the vertical, and the dipole is horizontal, so $\overrightarrow{\hat{p}} \cdot \vec{E}=0$. If $\beta$ were changed slightly, or the dipole displaced slightly from the horizontal, $G_{0}$ would not change much over most of the beam, but it would develop a deep minimum close to the boundary and then rise sharply to infinity at the boundary.

(iv) The magnetic field has a pronounced "pulling" effect tending to dominate the alignment of features in the polar diagram. This is perhaps most evident in figure 2 when $\chi_{p}=45^{\circ}$; as $\tau_{p}$ varies from $0^{\circ}$ to $90^{\circ}$, the continuous variation of the gain distribution is evident and the features are rotated by appreciably less than $90^{\circ}$ in $\tau_{0}$. Again, in figure 3 when $\tau_{0}=180^{\circ}$, the zero of gain moves from the edge of the beam to the center as $\beta$ varies from $0^{\circ}$ to $90^{\circ}$.

We are indebted to Mrs. Susan L. Bloch and to Melvin $G$. Whybra for assistance with the computations and preparation of illustrations. This work was supported by the National Aeronautics and Space Administration under grant NSG 572 and contract NAS 5-3099. 

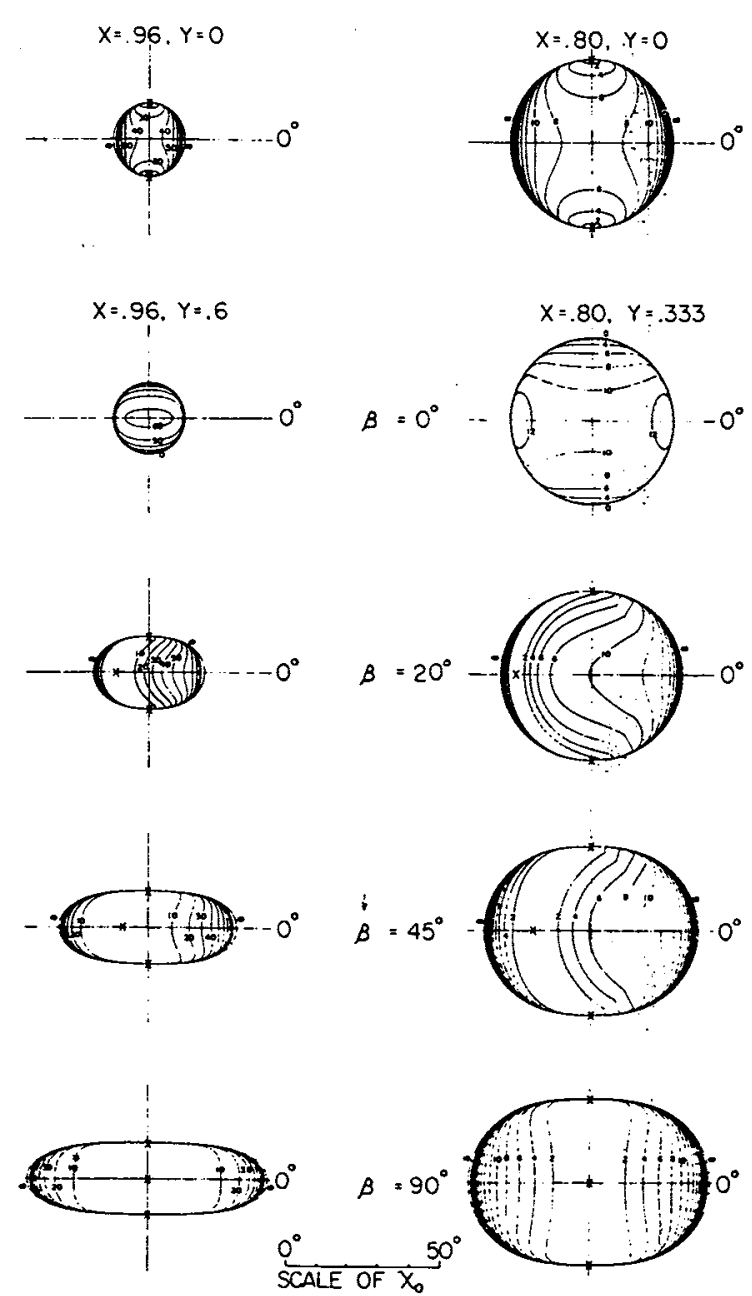

\section{References}

Budden, K. G. (1961a), The edge focusing of a radio signal received from outside the earth by an aerial within the ionosphere, Proc. Roy. Soc. London, Ser. A 263, No. 1315, 552-556.

Budden, K. G. (1961b), Radio Waves in the Ionosphere (University Press, Cambridge, England).

Budden, K. G., and J. Hugill (1964), The theory of the cosmic noise background signal received within the ionosphere, Proc. Roy. Soc. London, Ser. A 277, No. 1370. 365-384.

Daniell, G. J. (1964), Ionospheric focusing, Ann. Astrophys. 27, No. 6, 845-847. (Reprinted in Astronomical Observations from Space Vehicles, Proc. IAU Symp No. 23, Aug 1964, ed. J. L. Steinberg, 353-355 (Ann Astrophys. 92-Meudon, France).

Daniell, G. J. (1966), The effect of the curvature of the earth on radio-astronomical observations from satellites, J. Atmosph. Terr. Phys. 28, No. 1, 1-8.
FIGURE 3. Illustrating effect of varying the angle between geomagnetic field and vertical.

In each case, dipole orientation is $\chi_{p}=90^{\circ}, \tau_{p}=90^{\circ}$. For $x=0.8$, gain interval is 2 and maximum gain plotted is 40 ; for $z=0.96$, gain interval is 10 and maximum gain plotted is 100 . In addition, boundary of beam (gain $\infty$ or 0 ) is plotted in each case.

Haselgrove, C. B., J. Haselgrove, and R. C. Jennison (1961), Ray paths from a cosmic radio source to a satellite in orbit, Proc. Roy. Soc. London, Ser. A 261, No. 1306, 423-424.

Jennison, R. C. (1961), Proposed satellite techniques for perform. ing a high resolution survey of radio sky at medium wavelengths, J. Brit. IRE 22, No. 3, 205-208.

Yapagiannis, M. D., and G. R. Huguenin (1964), Ionospheric focusing in the presence of the earth's magnetic field, J. Geophys. Res. 69, No. $7,1307-1318$.

Smith, F. G. (1961), The possibility of observing features of galactic radio emission from a satellite-borne radio telescope, Monthly Notices Roy. Astronom. Soc. 122, No. 6, 527-534.

Weil, H., and D. Walsh (1967), Thermal radiation fields and antenna parameters in magnetoplasma, Radio Sci. 2 (New Series), No. 2, 225-239.<smiles>CCCC</smiles>

(Paper 3-9-449) 